\section{Disección coronaria espontánea post parto: reporte de un caso clínico y revisión de la literatura}

\author{
MARÍA LORENA JOFRÉ S. ${ }^{1}$, BERNARDITA GONZÁLEZ Z. ${ }^{\text {a }}$
}

\section{Spontaneous coronary artery dissection in the post partum: report of one case}

Coronary artery dissection is a rare cause of acute coronary syndrome. Most cases occur in women during the peripartum period, most likely influenced by hormonal changes, hemodynamic stress and modifications in the immune system during pregnancy. The pathogenesis of coronary artery dissection is unknown, hence numerous theories have been postulated such as pregnancy-related conditions, the presence of connective tissue disorders, trauma, etc. The clinical presentation ranges from asymptomatic patients to the whole spectrum of acute coronary syndrome manifestations. The management of these patients requires a multidisciplinary approach, with two options: medical therapy or an invasive approach, with percutaneous coronary intervention or coronary artery bypass graft surgery. The choice of treatment options depends on the hemodynamic status of the patient, the extension of the dissection and the myocardial territory at risk. In this case report we present a 38-year-old female who had a coronary artery dissection seven days postpartum. Coronary catheterization showed dissection of the left main coronary artery that extended until the circumflex artery. An intra-aortic balloon pump was installed and the patient then underwent coronary artery bypass graft surgery, with satisfactory results.

(Rev Med Chile 2014; 142: 391-394)

Key words: Coronary artery bypass; Coronary artery dissection, spontaneous; Postpartum period.

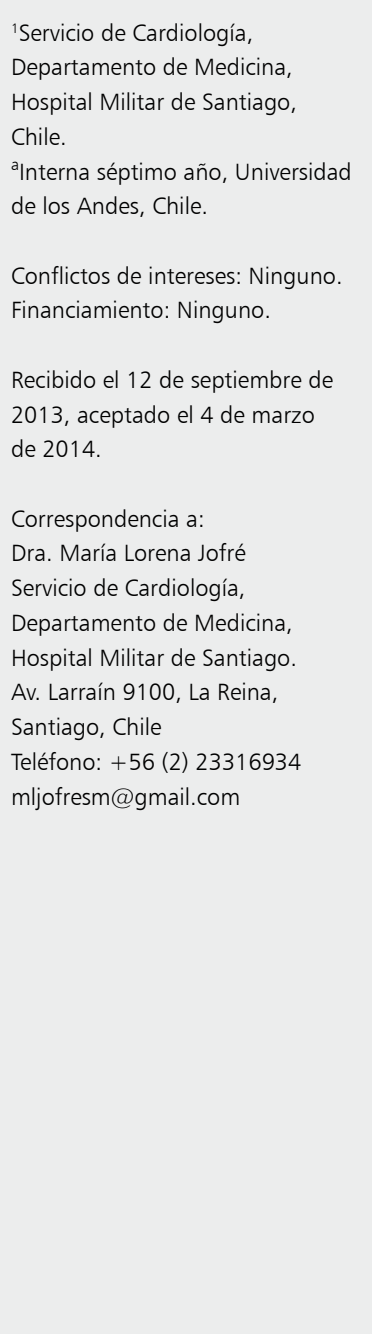

L a disección de las arterias coronarias puede ocurrir en forma espontánea o ser un evento secundario a traumatismo torácico, cirugía cardíaca, coronariografía, intervenciones coronarias o como una extensión de una disección aórtica ${ }^{1}$.

La disección de las arterias coronarias espontánea (DACE) es un evento infrecuente, que en algunas oportunidades puede asociarse a un síndrome coronario agudo ${ }^{2}$. Los casos de esta patología se observan con mayor frecuencia en mujeres, presentándose hasta en 30\% de las veces en el período periparto, probablemente potenciados por los cambios hormonales, estrés hemodinámico y modificaciones en el estado inmune propios de esta condición ${ }^{1,3}$.

La disección de las arterias coronarias genera la separación de las diferentes capas de la pared arterial, con la consecuente formación de un falso lumen. El plano de disección puede ubicarse entonces entre la íntima y la media, o entre la capa media y la adventicia. De esta manera, la hemorragia en el falso lumen-y subsiguiente trombosispuede comprimir el lumen verdadero, resultando en una obstrucción oclusiva o no-oclusiva del flujo sanguíneo ${ }^{1}$.

A continuación, presentamos un caso de disección espontánea de arterias coronarias. 


\section{Caso clínico}

Paciente de sexo femenino de 38 años, multípara de 5 , IMC $25,7 \mathrm{~kg} / \mathrm{m}^{2}$, puérpera de 7 días (parto por cesárea en otra institución), con antecedente de hipotiroidismo en tratamiento con levotiroxina $75 \mathrm{mcg} /$ día.

Consulta en un hospital general de Santiago por cuadro de $1 \mathrm{~h}$ de evolución caracterizado por dolor retroesternal opresivo de inicio súbito y máxima intensidad. Ingresa sin compromiso hemodinámico, afebril, con examen cardiopulmonar normal. Electrocardiograma (ECG) muestra infradesnivel del ST difuso (Figura 1). Se inicia manejo médico con aspirina, morfina y nitroglicerina sublingual; y se solicitan enzimas cardíacas y dímero D. Exámenes de ingreso evidencian: hemoglobina $14,9 \mathrm{~g} / \mathrm{dL}$, hematocrito $43,7 \%$, recuento de plaquetas $288.000 / \mathrm{uL}$, eosinófilos $2,9 \%$,
VHS $17 \mathrm{~mm} / \mathrm{h}$, creatinina $0,43 \mathrm{mg} / \mathrm{dL}$, troponina $0,45 \mathrm{ng} / \mathrm{ml}$, creatincinasa (CK) $72 \mathrm{U} / \mathrm{L}, \mathrm{Ck}-\mathrm{mb} 2,8$ U/L; dímero D $554 \mathrm{ng} / \mathrm{ml}$.

Se solicita AngioTAC de tórax por un dímero D elevado, que descarta tromboembolismo pulmonar.

Persiste con dolor y ECG de control evidencia QS en AVL, qR en DI y cambios en la amplitud de la onda R en V4-V6. Enzimas cardiacas de control con troponina $>50 \mathrm{ng} / \mathrm{ml}$, CK $3.800 \mathrm{U} / \mathrm{L}$ y Ck-mb $>300$ U/L. Coronariografía muestra disección de tronco de la arteria coronaria izquierda, que se extiende a la arteria circunfleja $(\mathrm{Cx})$ proximal y una rama marginal pequeña (Figura 2). Arteria descendente anterior (ADA) y coronaria derecha sin lesiones. Se instala balón de contrapulsación aórtica y se traslada a cirugía, donde se realiza bypass con arteria mamaria interna (LIMA) a ADA y puente safeno a lateral $\mathrm{Cx}$, sin incidentes.
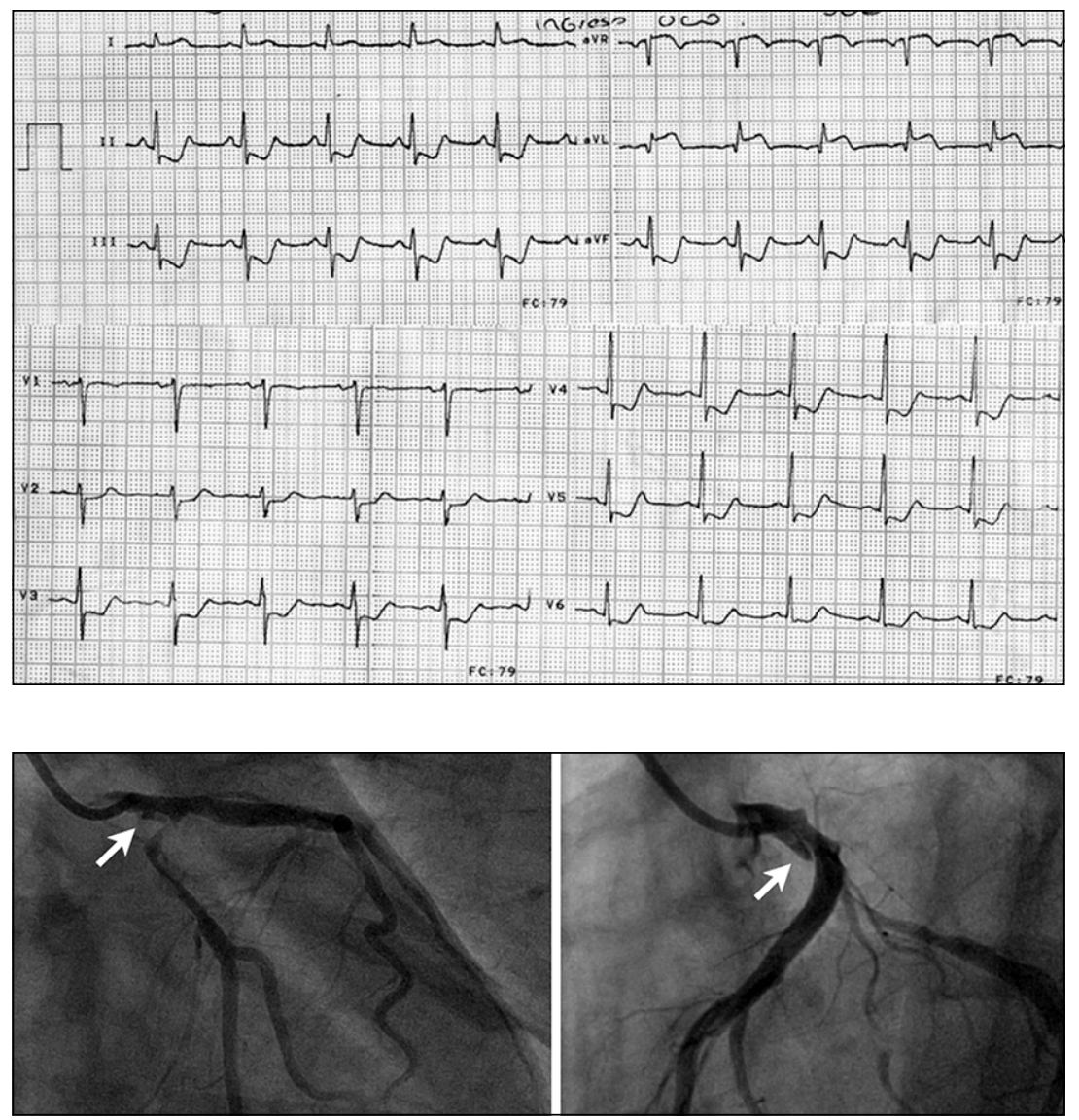

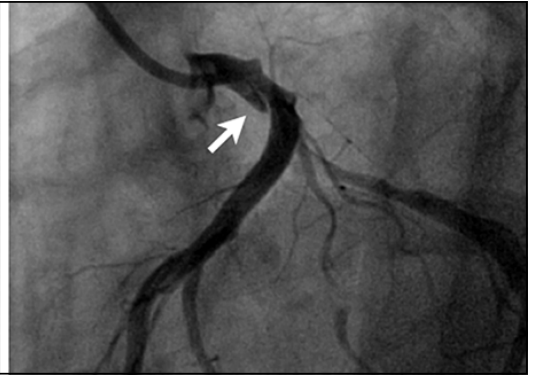

Figura 1. Electrocardiograma de 12 derivaciones donde se aprecia infradesnivel del segmento ST difuso, con supradesnivel del ST en aVL.

Figura 2. Coronariografía donde se aprecia flap de disección del tronco de la arteria coronaria izquierda (flecha blanca). 
Evoluciona favorablemente en el post operatorio, con retiro de drogas vasoactivas y balón de contrapulsación a las $48 \mathrm{~h}$.

Ecocardiograma de control con acinesia no adelgazada anterolateral con fracción de eyección de $42 \%$. Se optimiza control farmacológico de insuficiencia cardíaca con bisoprolol $1,25 \mathrm{mg}$ al día, espironolactona $25 \mathrm{mg}$ al día, enalapril $5 \mathrm{mg}$ cada $12 \mathrm{~h}$ y atorvastatina $40 \mathrm{mg}$ al día. Se traslada al quinto día post cirugía a otro centro por motivos previsionales.

Se controla en policlínico 15 días después del alta, encontrándose en buenas condiciones generales, asintomática, sin angina, en capacidad funcional I, con el tratamiento ya descrito, sin lactancia materna.

\section{Discusión}

La disección coronaria espontánea es una causa poco frecuente de síndrome coronario agudo (SCA). Tiene una incidencia global en coronariografías de 0,28 a $1,1 \% \%^{2,3,4}$. Es más frecuente en mujeres, de las cuales hasta en $30 \%$ de los casos se presenta en el período periparto (10\% a finales del embarazo y $20 \%$ en el post parto), probablemente influenciado por los cambios propios de esta condición ${ }^{1,3}$.

La arteria descendente anterior (ADA) es el vaso que con mayor frecuencia se diseca, sin embargo, también se han descrito casos de menor prevalencia en la arteria coronaria derecha, tronco de la arteria coronaria izquierda y circunfleja ${ }^{1,2,5}$. Una revisión de casos de DACE asociada al embarazo entre los años 1999 y 2008, publicada por Appleby y colaboradores, destaca que la mayoría de los casos ocurrieron en el período post parto, siendo la ADA la más frecuentemente afectada, con afectación de múltiples vasos en 30\% de los casos ${ }^{3}$.

La patogenia de esta condición aún no está del todo establecida, sin embargo, existen estudios que plantean que puede estar asociada al embarazo, uso de anticonceptivos orales, drogas ilícitas tales como la cocaína, ejercicio excesivo o secundaria a un trauma, manifestándose generalmente en el contexto de enfermedades del tejido conectivo tales como el síndrome de Marfan y Ehlers Danlos ${ }^{1}$. Por otra parte, existen estudios que establecen una asociación entre la disección coronaria y la presencia de eosinófilos, cuyos gránulos contienen numerosas sustancias líticas tales como colagenasa, peroxidasa y fosfatasa ácida, entre otras. Estas sustancias pueden romper las capas medias de la adventicia y provocar la disección. Durante el trabajo de parto y el periparto, los eosinófilos infiltran el útero, aumentando los niveles de colagenasa plasmática. Estudios animales muestran que tanto el estrógeno como la progesterona, ambos elevados durante el embarazo y el período periparto, pueden estimular a los eosinófilos a liberar estos gránulos con sustancias líticas. Por otra parte, durante el embarazo se producen cambios microestructurales en las fibras elásticas y colágenas de la túnica media de la aorta, que también se observan en las arterias coronarias, que pueden estar dadas por factores hormonales y hemodinámicos, contribuyendo a la predisposición de disección en el período periparto ${ }^{1-3,5}$. Todos estos cambios estructurales en las paredes arteriales y el estrés hemodinámico pueden persistir por un período de hasta 6 meses post parto ${ }^{3,5}$.

La presentación clínica suele ser variable de paciente a paciente, sin embargo, se ha descrito que el dolor torácico sería la manifestación más común ${ }^{6}$, el cual puede asociarse a infarto agudo al miocardio, shock cardiogénico o muerte súbita ${ }^{1-5}$. La severidad clínica va a depender entonces de la extensión de la disección y del territorio involucrado, con presentaciones que abarcan desde la ausencia de síntomas a todo el espectro de los $\mathrm{SCA}^{3}$.

Aun cuando la coronariografía es usada en forma rutinaria como método diagnóstico en los casos de sospecha de disección coronaria, se ha visto que la tomografía computada cardiaca puede proveer información complementaria, constituyendo un buen método de seguimiento de estas lesiones. El aspecto típico de la DACE en la coronariografía es la imagen de medio de contraste separado entre dos capas, con un retardo del clearance del contraste en el falso lumen ${ }^{1,3}$. Sin embargo, no siempre es posible identificar estas lesiones angiográficamente, por lo cual se hace necesario emplear otros métodos diagnósticos tales como el ultrasonido intravascular (IVUS) para determinar con mayor precisión la anatomía coronaria ${ }^{3}$.

El tratamiento de la DACE debe ser multidisciplinario, con dos grandes alternativas: terapia médica conservadora o un manejo invasivo a partir de cirugía o angioplastia coronaria percutánea (ACP). Dentro de los factores a considerar en la 
elección, encontramos el estado hemodinámico del paciente, el sitio de disección, el número de vasos comprometidos, el territorio involucrado y la extensión de miocardio en riesgo ${ }^{1-5}$.

La terapia médica durante el post parto implica el uso de antiplaquetarios (aspirina y clopidogrel) asociado a inhibidores de la enzima convertidora de angiotensina y beta bloqueadores. Sin embargo, es de importancia considerar que el uso de clopidogrel no está recomendado durante la lactancia ${ }^{3,5}$.

La ACP con el uso de stent ha demostrado ser una aproximación efectiva en estos casos, restaurando el flujo en el lumen verdadero, reduciendo la isquemia y sellando la disección ${ }^{1}$. Sin embargo, aún no está claro si el uso de stents liberadores de drogas sería de mayor beneficio en estas pacientes que el uso de stent metálicos ${ }^{5}$.

La cirugía con bypass coronario es considerada en los casos severos con enfermedad de múltiples vasos, presentaciones con inestabilidad hemodinámica o casos en los que hay fracaso de la revascularización ${ }^{2,5}$. En el caso de nuestra paciente, dada la ubicación y extensión de la disección, se optó por esta forma de terapia con un resultado exitoso.

El pronóstico a largo plazo en los pacientes que sobreviven a la fase aguda de la DACE es bueno, con una baja tasa de recurrencia del evento o de $\mathrm{SCA}^{4}$. La mortalidad asociada a esta condición se estima en $3 \%(0-4 \%)$ y se calcula una tasa de sobrevida de $95 \%$ en un plazo de 2 años ${ }^{1}$.

En conclusión, la DACE es una entidad poco frecuente, que se ve con mayor frecuencia en mujeres durante el embarazo y post parto, asociada a una mayor morbilidad y mortalidad. Se requiere de un alto índice de sospecha y coronariografía para su diagnóstico. El manejo va a depender entonces de la estabilidad de la paciente, así como también del tamaño de disección y el territorio afectado. En base a estos parámetros, se optará por un manejo médico o intervencional, con ACP o cirugía.

\section{Referencias}

1. Vrints C. Spontaneous coronary artery dissection. Heart 2010; 96: 801-8.

2. Shahzad K, Cao, L, Tun Q, Waddy J, Khan N, Nekkanti R. Post partum spontaneous dissection of the first obtuse marginal branch of theleftcircumflexcoronaryarterycausingacutecoronarysyndrome: a case report and literaturereview. Journal of Medical Case Reports 2013; 7: 82 .

3. Appleby C, Barolet A, Horlick E. Contemporary management of pregnancy-related coronary artery dissection: A single-centre experience and literature review. Exp Cln Cardiol 2009; 14 (1): e8-e16.

4. Tweet M, Hayes SN, Pitta SR, Simari RD, Lerman A, Lerman RJ, et al. Clinical Features, Management, and Prognosis of Spontaneous Coronary Artery Dissection. Circulation 2012; 126: 579-88.

5. Sheikh A, O'Sullivan M. Pregnancy-related Spontaneous Coronary Artery Dissection: Two Case Reports and a Comprehensive Review of Literature. HeartViews 2012; 13: 53-65.

6. Cenkowski M, daSilva M, Bordun K, Hussain F, Kirkpatrick I, Jassal D. Spontaneous dissection of the coronary and vertebral arteries post-partum: case report and review of the literature. BMC Pregnancy and Child birth 2012; 12: 122.

7. Kealey A. Coronary artery disease and myocardial infarction inpregnancy: A reviewof epidemiology, diagnosis, and medical and surgical management. Can J cardiol 2010; 26 (6): e185-9. 